\title{
Beyerane Derivatives and a Sesquiterpene Dimer from Japanese Cypress (Chamaecyparis obtusa)
}

\author{
Hui Yuan Gao, ${ }^{a}$ Li Jun Wu ${ }^{a}$ Norio Muto, ${ }^{b}$ Hiroyuki Fuchino, ${ }^{c}$ Takahisa Nakane, ${ }^{d}$ Osamu Shirota,${ }^{e}$ \\ Toshikazu SANO, ${ }^{f}$ and Masanori KuROYANAGI $*, b$ \\ ${ }^{a}$ School of Traditional Chinese Medicines, Shenyang Pharmaceutical University; 110016, No. 103, Wenhua Road, Shenhe \\ Shenyang, People's Republic of China: ${ }^{b}$ Faculty of Life and Environmental Sciences, Prefectural University of Hiroshima; \\ 562 Nanatsuka, Shobara, Hiroshima 727-0023, Japan: ${ }^{c}$ Research Center for Medicinal Plant Resources, National \\ Institute of Biomedical Innovation; 1 Hachimandai, Tsukuba, Ibaraki 305-0843, Japan: ${ }^{\text {Showa Pharmaceutical }}$ \\ University; 3-3165 Higashi-Tamagawagakuen, Machida, Tokyo 194-8543, Japan: ${ }^{e}$ Faculty of Pharmaceutical Sciences at \\ Kagawa Campus, Tokushima Bunri University; 1314-1 Shido, Sanuki, Kagawa 769-2193 Japan: and ${ }^{f}$ Hiroshima \\ Prefectural Forestry Research Center; 168-1 Tokaichi, Miyoshi, Hiroshima 728-0015, Japan.
}

Received March 4, 2008; accepted April 23, 2008

In the course of studies on biological active constituents from woody plants, we previously reported the isolation of many lignan derivatives as neurite outgrowth-promoting compounds from an ethyl acetate soluble fraction of Japanese Cypress (Chamaecyparis obtusa). Further chemical investigation on the residual parts of the ethyl acetate soluble fraction of a methanol extract of Japanese Cypress resulted in the isolation of four new beyerene type derivatives and a novel sesquiterpene dimer formed between cryptomeridiol and hinokiic acid. Their structures were elucidated as 18- $O$ - $(Z)$ - $p$-coumaroylbeyer-15-ene-18-ol $(1)$, 18- $O$ - $(E)$ - $p$-coumaroylbeyer-15ene-18-ol (2), 18- $O$ - $(E)$-p-coumaroylbeyer-15-ene-11 $\beta, 18$-diol (3), 18- $O$-( $Z$ )-p-coumaroylbeyer-15-ene-11 $\beta, 18$-diol (4) and ent-cryptomeridiol-4-yl-hinokiiate (5) by ${ }^{1} \mathrm{H}-\mathrm{NMR},{ }^{13} \mathrm{C}-\mathrm{NMR}$, 2D-NMR, and HR-MS spectral analysis.

Key words Chamaecyparis obtusa; Cupressaceae; beyerene derivative; sesquiterpene dimer

Social problems resulting from aging and degeneration of brain function have drawn attention around the world and especially in Japan. Biologically active constituents from plant resources are expected to suppress the development of these problems. We have studied various biologically active constituents from woody plants collected in the Chugoku area of Japan. $^{1-3)}$ In our previous work, the ethyl acetate (EtOAc) soluble fraction of Japanese Cypress (Chamaecyparis obtusa) showed neurite outgrowth-promoting activity on neuronal PC12 cells, and we isolated two novel compounds, 9$O$-acetoxydihydrosesamin and a lignan-sesquiterpene conjugate, together with eleven known compounds, and some of the compounds showed potent neurite outgrowth activity. ${ }^{4)}$ In order to find more new constituents from this fraction, further chemical investigations of the residual fraction were carried out and four new beyerene type derivatives $\mathbf{1}-\mathbf{4}$ (named obtsurins $\mathrm{A}-\mathrm{D}$ ) and a new sesquiterpene dimer ent-cryptomeridiol-4-yl-hinokiate (5), were obtained. Their structures were determined by means of spectral analysis including 2DNMR and high-resolution (HR)-MS. This paper deals with the isolation and structural elucidation of the new compounds from C. obtusa.

\section{Results and Discussion}

Compound 1 was obtained as colorless needles; mp 115$117^{\circ} \mathrm{C}$. The molecular formula of 1 was determined to be $\mathrm{C}_{29} \mathrm{H}_{38} \mathrm{O}_{3}$ based on the molecular ion peak at $\mathrm{m} / \mathrm{z} 434.2823$ $[\mathrm{M}]^{+}$in the HR-EI-MS. The IR spectrum of 1 showed absorption bands at 3340, 2931, 1632, 1687, 1606, and $1514 \mathrm{~cm}^{-1}$ ascribable to the hydroxyl, olefin, conjugated carbonyl, and phenyl functions. The ${ }^{1} \mathrm{H}-\mathrm{NMR}$ spectrum of $\mathbf{1}$ showed the presence of one $(Z)$-p-coumaroyl moiety $\left[\delta_{\mathrm{H}} 5.87\right.$ $(1 \mathrm{H}, \mathrm{d}, J=12.6 \mathrm{~Hz}), 6.86(1 \mathrm{H}, \mathrm{d}, J=12.6 \mathrm{~Hz}), 7.62(2 \mathrm{H}, \mathrm{d}$, $J=8.4 \mathrm{~Hz}), 6.79(2 \mathrm{H}, \mathrm{d}, J=8.4 \mathrm{~Hz})]$, three singlet methyl groups $\left[\delta_{\mathrm{H}} 0.77(3 \mathrm{H}, \mathrm{s}), 0.84(3 \mathrm{H}, \mathrm{s}), 0.99(3 \mathrm{H}, \mathrm{s})\right]$, and two characteristic olefinic protons coupled with each other $\left[\delta_{\mathrm{H}}\right.$ $5.45(1 \mathrm{H}, \mathrm{d}, J=5.4 \mathrm{~Hz}), 5.67(1 \mathrm{H}, \mathrm{d}, J=5.4 \mathrm{~Hz})]$ for $\mathrm{H}-15$ and $\mathrm{H}-16$ of beyer-15-ene skeleton, and one oxygenated methylene protons $\left[\delta_{\mathrm{H}} 3.72(1 \mathrm{H}, \mathrm{d}, J=11.4 \mathrm{~Hz}), 3.91(1 \mathrm{H}, \mathrm{d}\right.$, $J=11.4 \mathrm{~Hz}$ )] along with other alkyl protons. The ${ }^{13} \mathrm{C}-\mathrm{NMR}$ spectrum of 1 gave 27 carbon signals and showed the presence of a $p$-coumaroyl moiety $\left(\delta_{\mathrm{C}} 115.0 \times 2,132.5 \times 2,117.3\right.$, $127.5,143.4,156.9,166.9)$, two characteristic olefinic carbons $\left(\delta_{\mathrm{C}} 135.1,136.4\right)$ for $\mathrm{C}-15$ and $\mathrm{C}-16$ of beyer-15-ene skeleton, three methyl carbons $\left(\delta_{\mathrm{C}} 24.9,17.7,15.5\right)$, and an oxymethyl group $\left(\delta_{\mathrm{C}} 73.0\right)$. These characteristic olefinic ${ }^{1} \mathrm{H}-$ NMR signal ${ }^{5-8)}$ and ${ }^{13} \mathrm{C}-\mathrm{NMR}$ signal ${ }^{5,7,8)}$ showed the presence of beyer-15-ene skeleton in the structure. So compound 1 should be composed of a (Z)- $p$-coumaroyl and a beyer-15ene units through an ester linkage. This expectation and the position of ( $Z$ )- $p$-coumaroyl linkage were confirmed by HMBC analysis of 1 (Fig. 2). In the HMBC spectrum of $\mathbf{1}$, $\mathrm{H}_{3}-19\left(\delta_{\mathrm{H}} 0.84\right)$ showed correlations to $\mathrm{C}-18, \mathrm{C}-3$ and $\mathrm{C}-5$ $\left(\delta_{\mathrm{C}} 73.0,35.9,49.8\right)$, and $\mathrm{H}-18\left(\delta_{\mathrm{H}} 3.72, \mathrm{~d}, J=11.4 \mathrm{~Hz}, 3.91\right.$, $\mathrm{d}, J=11.4 \mathrm{~Hz}$ ) showed correlations to $\mathrm{C}-5, \mathrm{Me}-19$, and $\mathrm{C}-9^{\prime}$ $\left(\delta_{\mathrm{C}} 49.8,17.7,166.9\right)$. Other HMBC correlations are shown in Fig. 2. This data indicates that the $(Z)$ - $p$-coumaroyl group connects to a hydroxymethyl at C-4 through an ester bond. Alkaline hydrolysis of $\mathbf{1}$ yielded $\mathbf{1 a}$ and $p$-coumaric acid. The ${ }^{1} \mathrm{H}-\mathrm{NMR},{ }^{13} \mathrm{C}-\mathrm{NMR}, 2 \mathrm{D}-\mathrm{NMR}$, and MS data showed that 1a was beyer-15-ene-18-ol. $\left.{ }^{9}\right)$ The relative stereochemistry of $\mathbf{1}$ was determined by ROESY analysis (Fig. 2). NOE correlations of $\mathrm{H}_{3}-20$ to $\mathrm{H}-15$ and $\mathrm{H}_{3}-19 ; \mathrm{H}_{3}-19$ to $\mathrm{H}-18$ and $\mathrm{H}_{3}-20$; $\mathrm{H}-18$ to $\mathrm{H}-5$ and $\mathrm{H}_{3}-19$; and $\mathrm{H}_{3}-17$ to $\mathrm{H}-14$ and $\mathrm{H}-16$ were observed. Thus, the relative configuration of $\mathbf{1}$ was determined as shown in Fig. 1. The absolute configuration of 1a was determined from an opposite optical rotation of $\mathbf{1 a}\left([\alpha]_{\mathrm{D}}\right.$ $\left.-23.2^{\circ}\right)$ to the reported optical rotation data $\left([\alpha]_{\mathrm{D}}+33.9^{\circ}\right)^{9)}$ of the ent-isomer. Thus, the structure of 1 was determined to be $18-O-(Z)-p$-coumaroylbeyer-15-ene-18-ol and was named 
obtsurin A.

Compound 2 was obtained as an amorphous solid. The molecular formula of $\mathbf{2}$ was determined to be $\mathrm{C}_{29} \mathrm{H}_{38} \mathrm{O}_{3}$ based on the molecular ion peak at $m / z 434.2832[\mathrm{M}]^{+}$in the HREI-MS. The ${ }^{1} \mathrm{H}$ - and ${ }^{13} \mathrm{C}-\mathrm{NMR}$ spectral data of 2 showed similar signal patterns to those of $\mathbf{1}$ (Tables 1,2 ) except for a small difference in the $p$-coumaroyl moiety. The ${ }^{1} \mathrm{H}-\mathrm{NMR}$ spectrum of 2 showed the presence of an $(E)$-p-coumaroyl unit $\left[\delta_{\mathrm{H}} 6.32(1 \mathrm{H}, \mathrm{d}, J=16.2 \mathrm{~Hz}), 7.62(1 \mathrm{H}, \mathrm{d}, J=16.2 \mathrm{~Hz})\right.$, $7.45(2 \mathrm{H}, \mathrm{d}, J=9.0 \mathrm{~Hz}), 6.86(2 \mathrm{H}, \mathrm{d}, J=9.0 \mathrm{~Hz})]$, which indicated 2 was a $E$-isomer of 1 . The $2 \mathrm{D}-\mathrm{NMR}$ spectra, including HMQC, HMBC, and the ${ }^{1} \mathrm{H}-{ }^{1} \mathrm{H}$ COSY showed the same result as those of 1. NOESY analysis of $\mathbf{2}$ also showed the same result as that of $\mathbf{1}$. Alkaline hydrolysis of $\mathbf{2}$ gave 1a and $(E)$-p-coumaric acid. The optical rotation of $\mathbf{1 a}\left([\alpha]_{\mathrm{D}}\right.$ $\left.-19.7^{\circ}\right)$ from 2 was identified with $\mathbf{1 a}\left([\alpha]_{D}-23.2^{\circ}\right)$ from $\mathbf{1}$.
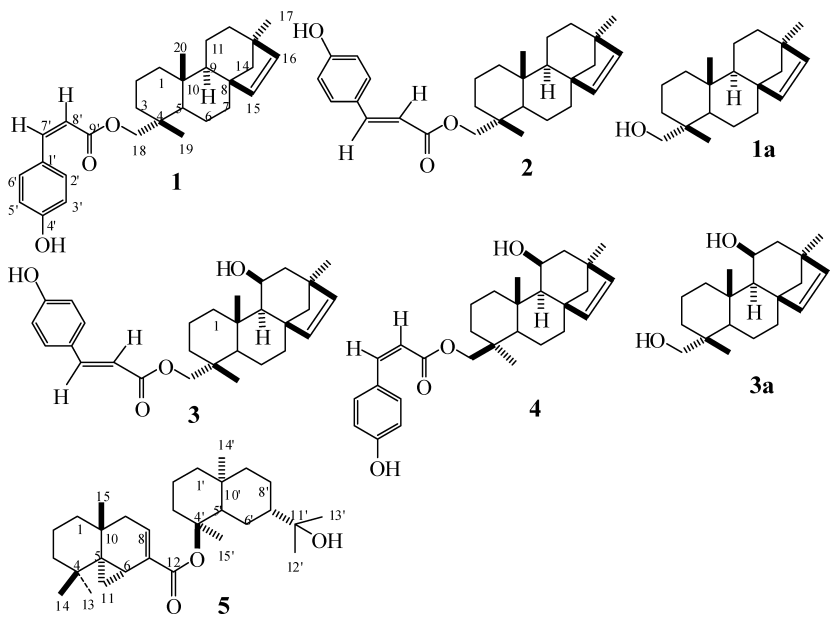

Fig. 1. Structures of $\mathbf{1}-\mathbf{5}, \mathbf{1} \mathbf{a}$ and $\mathbf{3 a}$
Thus, the structure of 2 was determined to be $18-O-(E)-p$ coumaroylbeyer-15-ene-18-ol and was named obtsurin B.

Compound 3 was obtained as a colorless amorphous solid. The molecular formula of 3 was determined as $\mathrm{C}_{29} \mathrm{H}_{38} \mathrm{O}_{4}$ based on the molecular ion peak at $m / z 450.2784$ in the HREI-MS. The ${ }^{1} \mathrm{H}-\mathrm{NMR}$ spectrum of $\mathbf{3}$ showed the existence of a $(E)$-p-coumaroyl moiety $\left[\delta_{\mathrm{H}} 6.32(1 \mathrm{H}, \mathrm{d}, J=15.5 \mathrm{~Hz}), 7.62\right.$ $(1 \mathrm{H}, \mathrm{d}, J=15.5 \mathrm{~Hz}), 6.85(2 \mathrm{H}, \mathrm{d}, J=8.5 \mathrm{~Hz}), 7.45(2 \mathrm{H}, \mathrm{d}$, $J=8.5 \mathrm{~Hz})]$, three singlet methyl groups $\left[\delta_{\mathrm{H}} 1.23(3 \mathrm{H}, \mathrm{s})\right.$, $1.06(3 \mathrm{H}, \mathrm{s}), 0.96(3 \mathrm{H}, \mathrm{s})]$, two olefinic protons coupled to each other $\left[\delta_{\mathrm{H}} 6.23(1 \mathrm{H}, \mathrm{d}, J=6.0 \mathrm{~Hz}), 5.79(1 \mathrm{H}, \mathrm{d}\right.$, $J=6.0 \mathrm{~Hz}$ )] characteristic for an beyer-15-ene skeleton, an oxygenated methylene group $\left[\delta_{\mathrm{H}} 3.98(1 \mathrm{H}, \mathrm{d}, J=10.5 \mathrm{~Hz})\right.$, $3.77(1 \mathrm{H}, \mathrm{d}, J=10.5 \mathrm{~Hz})]$, and an oxygenated methine group $\left(\delta_{\mathrm{H}} 4.23,1 \mathrm{H}, \mathrm{dt}, J=11.5,5.0 \mathrm{~Hz}\right)$, which was coupled with a hydroxyl proton $\left(\delta_{\mathrm{H}} 2.39,1 \mathrm{H}, \mathrm{d}, J=12.0 \mathrm{~Hz}\right)$. The ${ }^{13} \mathrm{C}-\mathrm{NMR}$ spectrum of 3 (Table 2) showed a similar signal pattern to that of $\mathbf{2}$ except for the presence of an additional hydroxyl group. These data indicated that $\mathbf{3}$ was a hydroxy derivative of 2 . In the ${ }^{1} \mathrm{H}-{ }^{1} \mathrm{H}$ COSY spectrum of $3 \mathrm{H}-9\left(\delta_{\mathrm{H}} 1.29,1 \mathrm{H}, \mathrm{d}\right.$, $J=5.0 \mathrm{~Hz})$ and $\mathrm{H}-12_{\mathrm{a}}\left(\delta_{\mathrm{H}} 1.80,1 \mathrm{H}, \mathrm{dd}, J=14.0,5.0 \mathrm{~Hz}\right)$ showed correlations to $\mathrm{H}-11$. In the HMBC spectrum of $\mathbf{3}$, $\mathrm{H}-9\left(\delta_{\mathrm{H}} 1.29,1 \mathrm{H}, \mathrm{d}, J=5.0 \mathrm{~Hz}\right)$ exhibited long-range correlations with $\mathrm{C}-11\left(\delta_{\mathrm{C}} 73.2\right)$. Other HMBC correlations in $\mathbf{3}$ are showed in Fig. 2. Alkaline hydrolysis of $\mathbf{3}$ gave $\mathbf{3 a}$ and $(E)-p$ coumaric acid. The molecular formula of $\mathbf{3 a}$ was determined to be $\mathrm{C}_{20} \mathrm{H}_{32} \mathrm{O}_{2}$ based on the molecular ion at $m / z 304.2395$ in the HR-EI-MS. The ${ }^{1} \mathrm{H}-\mathrm{NMR},{ }^{13} \mathrm{C}-\mathrm{NMR}$, and 2D-NMR spectra of 3a showed that the structure of $\mathbf{3} \mathbf{a}$ had a beyer-15ene-diol structure. The position of the hydroxyl groups and relative configuration of $\mathbf{3 a}$ were examined by $2 \mathrm{D}-\mathrm{NMR}$ including HMBC and ROESY analyses. The HMBC spectrum of 3a showed correlations of $\mathrm{H}-11\left(\delta_{\mathrm{H}} 4.21\right.$, br t, $\left.J=5.4 \mathrm{~Hz}\right)$ to $\mathrm{C}-8, \mathrm{C}-9, \mathrm{C}-12$, and C-13 $\left(\delta_{\mathrm{C}} 48.3,59.7,45.3,42.5\right)$; H-18

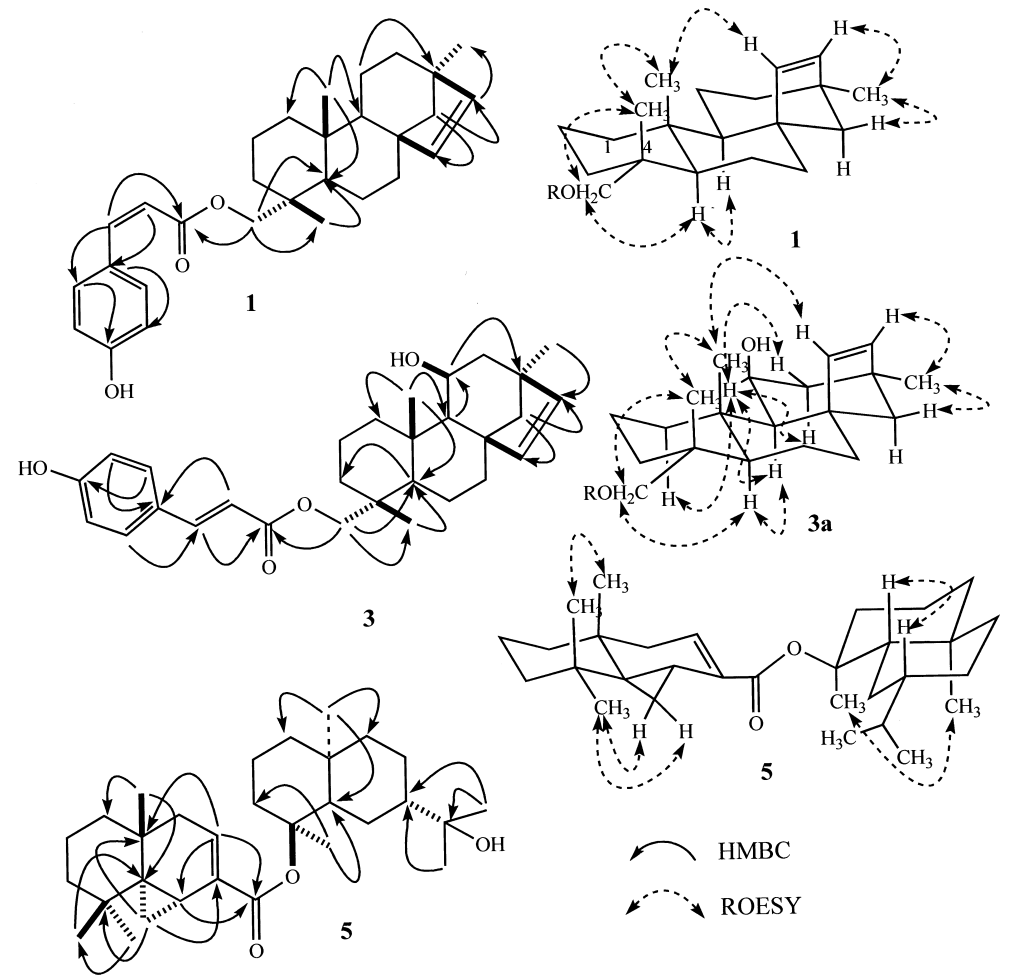

Fig. 2. Important HMBC Correlations of 1, $\mathbf{3}$ and 5, and ROESY Correlations of 1, 3a and $\mathbf{5}$ 
Table 1. ${ }^{1} \mathrm{H}-\mathrm{NMR}$ Spectrum of $\mathbf{1}-\mathbf{4}$ and $\mathbf{3 a}\left(\mathrm{CDCl}_{3}, 500 \mathrm{MHz}\right)^{a}$

\begin{tabular}{|c|c|c|c|c|c|}
\hline Position & 1 & 2 & 3 & 4 & $3 \mathbf{a}$ \\
\hline \multirow[t]{2}{*}{$\mathrm{H}-1$} & $0.81(1 \mathrm{H}, \mathrm{dd}, 12.6,3.6)$ & $0.86(1 \mathrm{H}, \mathrm{dd}, 12.6,3.0)$ & $1.06(1 \mathrm{H}$, overlap $)$ & & $1.02(1 \mathrm{H}, \mathrm{dt}, 3.6,13.0)$ \\
\hline & $1.59(1 \mathrm{H}$, overlap $)$ & $1.61(1 \mathrm{H}$, overlap $)$ & $1.99(1 \mathrm{H}$, brd, 12.5$)$ & $1.95(1 \mathrm{H}, \mathrm{brd}, 12.5)$ & $1.97(1 \mathrm{H}, \mathrm{dt}, 13.0,4.0)$ \\
\hline \multirow[t]{2}{*}{2} & $1.51(1 \mathrm{H}$, overlap $)$ & $1.55(1 \mathrm{H}$, overlap $)$ & $1.47(1 \mathrm{H}$, overlap $)$ & & $1.51(1 \mathrm{H}$, overlap $)$ \\
\hline & $1.40(1 \mathrm{H}$, overlap $)$ & $1.46(1 \mathrm{H}$, overlap $)$ & $1.66(1 \mathrm{H}$, overlap $)$ & & $1.62(1 \mathrm{H}, \mathrm{m})$ \\
\hline \multirow[t]{2}{*}{3} & $1.33(1 \mathrm{H}$, overlap $)$ & $1.38(1 \mathrm{H}$, overlap $)$ & $1.38(1 \mathrm{H}$, overlap $)$ & & $1.30(1 \mathrm{H}$, overlap $)$ \\
\hline & $1.29(1 \mathrm{H}$, overlap $)$ & $1.42(1 \mathrm{H}$, overlap $)$ & $1.48(1 \mathrm{H}$, overlap $)$ & & $1.38(1 \mathrm{H}, \mathrm{dt}, 4.2,13.8)$ \\
\hline 5 & $1.10(1 \mathrm{H}, \mathrm{dd}, 10.8,3.0)$ & $1.18(1 \mathrm{H}, \mathrm{dd}, 11.4,2.4)$ & $1.13(1 \mathrm{H}, \mathrm{dd}, 11.5,2.5)$ & $1.00(1 \mathrm{H}, \mathrm{dd}, 11.5,2.5)$ & $1.16(1 \mathrm{H}, \mathrm{dd}, 11.4,2.4)$ \\
\hline \multirow[t]{2}{*}{6} & $1.39(2 \mathrm{H}$, overlap $)$ & $1.41(1 \mathrm{H}$, overlap $)$ & $1.50(1 \mathrm{H}$, overlap $)$ & & $1.45(1 \mathrm{H}$, ovrlap $)$ \\
\hline & & $1.47(1 \mathrm{H}$, overlap $)$ & $1.55(1 \mathrm{H}$, overlap $)$ & & $1.49(1 \mathrm{H}$, overlap $)$ \\
\hline \multirow[t]{2}{*}{7} & $1.27(1 \mathrm{H}$, overlap $)$ & $1.34(1 \mathrm{H}, \mathrm{dd}, 13.8,4.8)$ & $1.38(1 \mathrm{H}$, overlap $)$ & & $1.40(1 \mathrm{H}$, overlap $)$ \\
\hline & $1.58(1 \mathrm{H}$, overlap $)$ & $1.63(1 \mathrm{H}$, overlap $)$ & $1.70(1 \mathrm{H}$, overlap $)$ & & $1.69(1 \mathrm{H}$, overlap $)$ \\
\hline 9 & $0.99(1 \mathrm{H}$, overlap $)$ & $1.04(1 \mathrm{H}$, overlap $)$ & $1.29(1 \mathrm{H}, \mathrm{d}, 5.0)$ & $1.23(1 \mathrm{H}, \mathrm{d}, 4.5)$ & $1.31(1 \mathrm{H}, \mathrm{d}, 4.8)$ \\
\hline \multirow[t]{2}{*}{11} & $1.25(1 \mathrm{H}$, overlap $)$ & $1.25(1 \mathrm{H}$, overlap $)$ & $4.23(1 \mathrm{H}, \mathrm{dt}, 11.5,5.0)$ & $4.21(1 \mathrm{H}, \mathrm{dt}, 11.5,5.0)$ & $4.21(1 \mathrm{H}, \mathrm{brt}, 5.4)$ \\
\hline & $1.50(1 \mathrm{H}$, overlap $)$ & $1.52(1 \mathrm{H}$, overlap $)$ & & & \\
\hline \multirow[t]{2}{*}{12} & $1.24(1 \mathrm{H}$, overlap $)$ & $1.27(1 \mathrm{H}$, overlap $)$ & $1.70(1 \mathrm{H}$, overlap $)$ & $1.70(1 \mathrm{H}, \mathrm{dd}, 14.5,2.0)$ & $1.71(1 \mathrm{H}, \mathrm{dd}, 13.8,5.4)$ \\
\hline & $1.29(1 \mathrm{H}$, overlap $)$ & $1.29(1 \mathrm{H}$, overlap $)$ & $1.80(1 \mathrm{H}, \mathrm{dd}, 14.0,5.0)$ & $1.79(1 \mathrm{H}, \mathrm{dd}, 14.5,5.0)$ & $1.79(1 \mathrm{H}, \mathrm{dd}, 13.8,2.4)$ \\
\hline \multirow[t]{2}{*}{14} & $1.01(1 \mathrm{H}, \mathrm{d}, 9.6)$ & $1.06(1 \mathrm{H}, \mathrm{d}, 10.2)$ & $1.22(1 \mathrm{H}, \mathrm{d}, 10.0)$ & & $1.22(1 \mathrm{H}, \mathrm{d}, 9.6)$ \\
\hline & $1.43(1 \mathrm{H}$, overlap $)$ & $1.44(1 \mathrm{H}$, overlap $)$ & $1.68(1 \mathrm{H}, \mathrm{d}, 10.0)$ & & $1.64(1 \mathrm{H}, \mathrm{dd}, 9.6,3.6)$ \\
\hline 15 & $5.67(1 \mathrm{H}, \mathrm{d}, 5.4)$ & $5.69(1 \mathrm{H}, \mathrm{d}, 5.4)$ & $6.23(1 \mathrm{H}, \mathrm{d}, 6.0)$ & $6.21(1 \mathrm{H}, \mathrm{d}, 5.5)$ & $6.22(1 \mathrm{H}, \mathrm{d}, 6.0)$ \\
\hline 16 & $5.45(1 \mathrm{H}, \mathrm{d}, 5.4)$ & $5.46(1 \mathrm{H}, \mathrm{d}, 5.4)$ & $5.79(1 \mathrm{H}, \mathrm{d}, 6.0)$ & $5.77(1 \mathrm{H}, \mathrm{d}, 5.5)$ & $5.78(1 \mathrm{H}, \mathrm{d}, 6.0)$ \\
\hline 17 & $0.99(3 \mathrm{H}, \mathrm{s})$ & $0.99(3 \mathrm{H}, \mathrm{s})$ & $1.06(3 \mathrm{H}, \mathrm{s})$ & $1.06(3 \mathrm{H}, \mathrm{s})$ & $1.06(3 \mathrm{H}, \mathrm{s})$ \\
\hline \multirow[t]{2}{*}{18} & $3.72(1 \mathrm{H}, \mathrm{d}, 11.4)$ & $3.79(1 \mathrm{H}, \mathrm{d}, 10.8)$ & $3.77(1 \mathrm{H}, \mathrm{d}, 10.5)$ & $3.71(1 \mathrm{H}, \mathrm{d}, 11.0)$ & $3.10(1 \mathrm{H}, \mathrm{d}, 10.8)$ \\
\hline & $3.91(1 \mathrm{H}, \mathrm{d}, 11.4)$ & $3.98(1 \mathrm{H}, \mathrm{d}, 10.8)$ & $3.98(1 \mathrm{H}, \mathrm{d}, 10.5)$ & $3.89(1 \mathrm{H}, \mathrm{d}, 11.0)$ & $3.39(1 \mathrm{H}, \mathrm{d}, 10.8)$ \\
\hline 19 & $0.84(3 \mathrm{H}, \mathrm{s})$ & $0.89(3 \mathrm{H}, \mathrm{s})$ & $0.96(3 \mathrm{H}, \mathrm{s})$ & $0.90(3 \mathrm{H}, \mathrm{s})$ & $0.84(3 \mathrm{H}, \mathrm{s})$ \\
\hline 20 & $0.77(3 \mathrm{H}, \mathrm{s})$ & $0.80(3 \mathrm{H}, \mathrm{s})$ & $1.23(3 \mathrm{H}, \mathrm{s})$ & $1.20(3 \mathrm{H}, \mathrm{s})$ & $1.22(3 \mathrm{H}, \mathrm{s})$ \\
\hline $2^{\prime}, 6^{\prime}$ & $7.62(2 \mathrm{H}, \mathrm{d}, 8.4)$ & $7.45(2 \mathrm{H}, \mathrm{d}, 9.0)$ & $7.45(2 \mathrm{H}, \mathrm{d}, 8.5)$ & $7.61(2 \mathrm{H}, \mathrm{d}, 8.5)$ & \\
\hline $3^{\prime}, 5^{\prime}$ & $6.79(2 \mathrm{H}, \mathrm{d}, 8.4)$ & $6.86(2 \mathrm{H}, \mathrm{d}, 9.0)$ & $6.85(2 \mathrm{H}, \mathrm{d}, 8.5)$ & $6.79(2 \mathrm{H}, \mathrm{d}, 8.5)$ & \\
\hline $7^{\prime}$ & $6.86(1 \mathrm{H}, \mathrm{d}, 12.6)$ & $7.62(1 \mathrm{H}, \mathrm{d}, 16.2)$ & $7.62(1 \mathrm{H}, \mathrm{d}, 15.5)$ & $6.85(1 \mathrm{H}, \mathrm{d}, 13.0)$ & \\
\hline $8^{\prime}$ & $5.87(1 \mathrm{H}, \mathrm{d}, 12.6)$ & $6.32(1 \mathrm{H}, \mathrm{d}, 16.2)$ & $6.32(1 \mathrm{H}, \mathrm{d}, 15.5)$ & $5.85(1 \mathrm{H}, \mathrm{d}, 13.0)$ & \\
\hline $\mathrm{OH}$ at $\mathrm{C}-11$ & & & $2.39(1 \mathrm{H}, \mathrm{d}, 12.0)$ & $2.35(1 \mathrm{H}, \mathrm{d}, 12.0)$ & \\
\hline
\end{tabular}

a) ${ }^{1} \mathrm{H}-\mathrm{NMR}$ assignments of $\mathbf{1} \mathbf{- 3}$ and $\mathbf{3 a}$ were obtained from studies of 2D-NMR, but the assignment of $\mathbf{4}$ was not perfect because of no $2 \mathrm{D}-\mathrm{NMR}$ data was obtained due to the low sample amount.

Table 2. ${ }^{13} \mathrm{C}-\mathrm{NMR}$ Data of $\mathbf{1}-\mathbf{4}$ and $\mathbf{3 a}\left(125 \mathrm{MHz}\right.$ in $\left.\mathrm{CDCl}_{3}\right)$

\begin{tabular}{|c|c|c|c|c|c|}
\hline Position & 1 & 2 & 3 & 4 & $3 a$ \\
\hline $\mathrm{C}-1$ & 38.5 & 38.6 & 39.2 & 39.1 & 39.1 \\
\hline 2 & 17.8 & 17.8 & 17.6 & 17.6 & 17.6 \\
\hline 3 & 35.9 & 36.0 & 35.9 & 35.8 & 35.1 \\
\hline 4 & 36.5 & 36.9 & 36.7 & 36.6 & 37.4 \\
\hline 5 & 49.8 & 50.0 & 50.3 & 50.0 & 49.0 \\
\hline 6 & 20.0 & 20.1 & 20.5 & 20.4 & 20.1 \\
\hline 7 & 36.9 & 36.7 & 36.7 & 36.6 & 36.6 \\
\hline 8 & 48.9 & 48.9 & 48.3 & 48.3 & 48.3 \\
\hline 9 & 52.7 & 52.8 & 59.9 & 59.8 & 59.7 \\
\hline 10 & 37.1 & 37.2 & 38.3 & 38.3 & 38.1 \\
\hline 11 & 20.1 & 20.2 & 69.3 & 69.3 & 69.1 \\
\hline 12 & 33.1 & 33.1 & 45.3 & 45.3 & 45.3 \\
\hline 13 & 43.6 & 43.6 & 42.6 & 42.6 & 42.5 \\
\hline 14 & 60.1 & 61.1 & 63.3 & 63.3 & 63.3 \\
\hline 15 & 135.1 & 135.1 & 140.9 & 140.9 & 141.3 \\
\hline 16 & 136.4 & 136.4 & 141.4 & 141.4 & 140.8 \\
\hline 17 & 24.9 & 24.9 & 24.7 & 24.8 & 24.7 \\
\hline 18 & 73.0 & 73.1 & 73.2 & 73.2 & 72.2 \\
\hline 19 & 17.7 & 17.7 & 18.1 & 18.1 & 18.0 \\
\hline 20 & 15.5 & 15.5 & 18.7 & 18.7 & 18.7 \\
\hline $1^{\prime}$ & 127.5 & 127.2 & 127.3 & 127.6 & \\
\hline $2^{\prime}, 6^{\prime}$ & 132.5 & 130.0 & 130.0 & 132.2 & \\
\hline $3^{\prime}, 5^{\prime}$ & 115.0 & 115.8 & 115.9 & 115.1 & \\
\hline $4^{\prime}$ & 156.9 & 157.7 & 157.7 & 156.7 & \\
\hline $7^{\prime}$ & 143.4 & 144.2 & 144.3 & 143.3 & \\
\hline $8^{\prime}$ & 117.3 & 115.7 & 115.8 & 117.4 & \\
\hline $9^{\prime}$ & 166.9 & 167.6 & 167.5 & 166.8 & \\
\hline
\end{tabular}

$\left(\delta_{\mathrm{H}} 3.39, \mathrm{~d}, J=10.8 \mathrm{~Hz}, 3.10, \mathrm{~d}, J=10.8 \mathrm{~Hz}\right)$ to $\mathrm{C}-3, \mathrm{C}-4, \mathrm{C}-$ 5 , and $\mathrm{C}-19\left(\delta_{\mathrm{C}} 35.1,37.4,49.0,18.0\right)$. NOE analysis showed correlations of $\mathrm{H}_{3}-19\left(\delta_{\mathrm{H}} 0.84,3 \mathrm{H}, \mathrm{s}\right)$ to $\mathrm{H}-18$ and $\mathrm{H}_{3}-20\left(\delta_{\mathrm{H}} 1.22,3 \mathrm{H}, \mathrm{s}\right) ; \mathrm{H}_{3}-20$ to $\mathrm{H}_{3}-19, \mathrm{H}-15\left(\delta_{\mathrm{H}} 6.22, \mathrm{~d}\right.$, $J=6.0 \mathrm{~Hz}) ; \mathrm{H}-9\left(\delta_{\mathrm{H}} 1.31, \mathrm{~d}, J=4.8 \mathrm{~Hz}\right)$ to $\mathrm{H}-5\left(\delta_{\mathrm{H}} 1.16,1 \mathrm{H}\right.$, dd, $J=11.4,2.4 \mathrm{~Hz})$ and $\mathrm{H}-11 ; \mathrm{H}-11$ to $\mathrm{H}-9$ and $\mathrm{H}-12\left(\delta_{\mathrm{H}}\right.$ 1.79 , dd, $13.8,5.4 \mathrm{~Hz}, 1.71,1 \mathrm{H}, \mathrm{dd}, J=13.8,5.4 \mathrm{~Hz}$ ); and $\mathrm{H}-$ $16\left(\delta_{\mathrm{H}} 5.78, \mathrm{~d}, J=6.0 \mathrm{~Hz}\right)$ to $\mathrm{H}_{3}-17\left(\delta_{\mathrm{H}} 1.06,3 \mathrm{H}, \mathrm{s}\right)$ and $\mathrm{H}-15$ as shown in Fig. 2. The configuration of the hydroxyl group at $\mathrm{C}-11$ was suggested to be $\beta$-axial from the coupling constant $(J=4.8 \mathrm{~Hz})$ of $\mathrm{H}-9$ of $\mathbf{3 a}$. This was confirmed from the NOE correlations of $\mathrm{H}-11$ to $\mathrm{H}-9, \mathrm{H}-12 \alpha, \mathrm{H}-12 \beta$ and $\mathrm{H}-1 \beta$ as shown in Fig. 2. These data indicated that the structure of 3a was determined to be beyer-15-ene-11 $\beta, 18$-diol. This compound has not been reported previously, and the stereochemistry of $\mathbf{3}$ was confirmed as shown in Fig. 1. The absolute configuration of $\mathbf{3}$ has not been confirmed, but should be supposed to be the same with as of $\mathbf{1}$, because both compounds were isolated from the same source. Based on the above analysis, compound 3 was established as $18-O-(E)-p$ coumaroylbeyer-15-ene-11 $\beta, 18$-diol and was named obtsurin C.

Compound 4 was obtained as an amorphous solid. The molecular formula of 4 was determined to be $\mathrm{C}_{29} \mathrm{H}_{38} \mathrm{O}_{4}$ based on the molecular ion peak at $\mathrm{m} / \mathrm{z} 450.2789$ in the HR-EI-MS. This molecular formula was identical with that of 3 . The ${ }^{1} \mathrm{H}-$ NMR data of 4 showed the presence of a $(Z)$-p-coumaroyl moiety $\left[\delta_{\mathrm{H}} 5.85(1 \mathrm{H}, \mathrm{d}, J=13.0 \mathrm{~Hz}), 6.85(1 \mathrm{H}, \mathrm{d}, J=\right.$ 
$13.0 \mathrm{~Hz}), 7.61(2 \mathrm{H}, \mathrm{d}, J=8.5 \mathrm{~Hz}), 6.79(2 \mathrm{H}, \mathrm{d}, J=8.5 \mathrm{~Hz})]$ and a beyer-15-ene-11 $\beta, 18$-diol structure. The ${ }^{13} \mathrm{C}-\mathrm{NMR}$ spectrum data of $\mathbf{4}$ were almost identical with those of $\mathbf{3}$ except for the ( $Z$ )- $p$-coumaroyl moiety (see Table 2 ). Thus, the structure of 4 was determined to be $18-O-(Z)-p$-coumaroylbeyer-15-ene-11 $\beta$,18-diol and was named obtsurin $\mathrm{D}$.

Compound 5 was isolated as colorless needles, mp 56$58^{\circ} \mathrm{C}$. The molecular formula of 5 was determined to be $\mathrm{C}_{30} \mathrm{H}_{46} \mathrm{O}_{3}$ based on the molecular ion peak at $\mathrm{m} / \mathrm{z} 456.3586$ $[\mathrm{M}]^{+}$in the HR-EI-MS. The IR spectrum of 5 showed absorption at $3454,2930,1703,1650,1470$, and $1385 \mathrm{~cm}^{-1}$ ascribable to the hydroxyl, olefin, carbonyl, and alkyl groups. The ${ }^{1} \mathrm{H}-\mathrm{NMR}$ spectrum of $\mathbf{5}$ showed the presence of a cyclopropane ring $\left[\delta_{\mathrm{H}} 0.67(1 \mathrm{H}, \mathrm{t}, J=4.8 \mathrm{~Hz}), 0.77(1 \mathrm{H}, \mathrm{dd}\right.$, $J=9.0,4.8 \mathrm{~Hz})]$, seven singlet methyl groups $\left[\delta_{\mathrm{H}} 0.67(3 \mathrm{H}\right.$, s), $0.93(3 \mathrm{H}, \mathrm{s}), 1.11(3 \mathrm{H}, \mathrm{s}), 1.17(3 \mathrm{H}, \mathrm{s}), 1.20(3 \mathrm{H}, \mathrm{s}), 1.21$ $(3 \mathrm{H}, \mathrm{s}) 1.48(3 \mathrm{H}, \mathrm{s})]$, and an olefinic proton $\left[\delta_{\mathrm{H}} 6.34(1 \mathrm{H}\right.$, brd, $J=6.0 \mathrm{~Hz}$ )] together with many alkyl proton signals (Table 3 ). The ${ }^{13} \mathrm{C}-\mathrm{NMR}$ spectrum of 5 gave 30 carbon peaks and showed the presence of a carboxyl group $\left(\delta_{\mathrm{C}} 166.1\right)$, two olefinic carbons $\left(\delta_{\mathrm{C}} 133.5,131.4\right)$, two oxygenated quaternary carbons $\left(\delta_{\mathrm{C}} 85.6,72.8\right)$, four nonoxygenated quaternary carbons $\left(\delta_{\mathrm{C}} 31.4,33.9,34.6,34.7\right)$, seven methyls $\left(\delta_{\mathrm{C}} 18.8\right.$, $19.1,26.8,27.1,27.2,28.3,29.0)$, and three methines $\left(\delta_{\mathrm{C}}\right.$ $16.6,49.9,52.2)$ among other signals. The ${ }^{13} \mathrm{C}-\mathrm{NMR}$ chemical shifts of 5 were almost same as the summation of the ${ }^{13} \mathrm{C}$ NMR spectral data of hinokiic acid and criptomeridiol (see

Table 3. The ${ }^{1} \mathrm{H}$ - and ${ }^{13} \mathrm{C}-\mathrm{NMR}$ Data of Compound 5 (in $\mathrm{CDCl}_{3},{ }^{1} \mathrm{H}-$ $\left.600 \mathrm{MHz},{ }^{13} \mathrm{C}-150 \mathrm{MHz}\right)$

\begin{tabular}{|c|c|c|c|c|}
\hline \multirow{2}{*}{ Position } & \multicolumn{2}{|r|}{5} & \multirow{2}{*}{$\frac{\begin{array}{c}\text { Hinokiic } \\
\text { acid }\end{array}}{\delta_{\mathrm{C}}}$} & \multirow{2}{*}{$\begin{array}{l}\text { Cripto- } \\
\text { meridiol } \\
\delta_{\mathrm{C}}\end{array}$} \\
\hline & $\delta_{\mathrm{C}}$ & $\delta_{\mathrm{H}}$ & & \\
\hline 1 & 36.9 & $1.14(1 \mathrm{H}$, overlap $), 1.28(1 \mathrm{H}$, overlap $)$ & 37.1 & \\
\hline 2 & 19.4 & $1.76(1 \mathrm{H}, \mathrm{qt}, 13.8,3.6), 1.45(1 \mathrm{H}, \mathrm{m})$ & 19.4 & \\
\hline 3 & 40.0 & $1.42(1 \mathrm{H}$, overlap $), 1.25(1 \mathrm{H}, \mathrm{m})$ & 40.1 & \\
\hline 4 & 33.9 & & 33.9 & \\
\hline 5 & 34.6 & & 34.7 & \\
\hline 6 & 16.8 & $2.04(1 \mathrm{H}, \mathrm{dd}, 9.0,4.8)$ & 16.6 & \\
\hline 7 & 133.5 & & 131.2 & \\
\hline 8 & 131.4 & $6.34(1 \mathrm{H}$, br d, 6.0$)$ & 135.6 & \\
\hline 9 & 41.4 & $\begin{array}{l}1.68(1 \mathrm{H}, \mathrm{dd}, 18.0,7.2) \\
1.89(1 \mathrm{H}, \mathrm{dd}, 18.0,2.4)\end{array}$ & 41.4 & \\
\hline 10 & 31.4 & & 31.5 & \\
\hline 11 & 11.4 & $0.67(1 \mathrm{H}, \mathrm{t}, 4.8), 0.77(1 \mathrm{H}, \mathrm{dd}, 9.0,4.8)$ & 11.5 & \\
\hline 12 & 166.1 & & 171.4 & \\
\hline 13 & 29.0 & $0.67(3 \mathrm{H}, \mathrm{s})$ & 26.8 & \\
\hline 14 & 26.8 & $1.11(3 \mathrm{H}, \mathrm{s})$ & 29.0 & \\
\hline 15 & 28.3 & $1.17(3 \mathrm{H}, \mathrm{s})$ & 28.3 & \\
\hline $1^{\prime}$ & 40.5 & $1.13(1 \mathrm{H}$, overlap $), 1.40(1 \mathrm{H}$, overlap $)$ & & 41.4 \\
\hline $2^{\prime}$ & 19.7 & $1.581 \mathrm{H}$, (overlap), $1.60(1 \mathrm{H}$, overlap) & & 18.0 \\
\hline $3^{\prime}$ & 38.1 & $2.75(1 \mathrm{H}$, br d, 10.5$), 1.66(1 \mathrm{H}$, overlap $)$ & & 41.3 \\
\hline $4^{\prime}$ & 85.6 & & & 72.1 \\
\hline $5^{\prime}$ & 52.2 & $1.66(1 \mathrm{H}$, overlap $)$ & & 51.6 \\
\hline $6^{\prime}$ & 22.2 & $1.92(1 \mathrm{H}$, br s $), 1.11(1 \mathrm{H}$, overlap $)$ & & 21.3 \\
\hline $7^{\prime}$ & 49.8 & $1.40(1 \mathrm{H}$, overlap $)$ & & 49.9 \\
\hline $8^{\prime}$ & 22.4 & $1.62(1 \mathrm{H}$, overlap $), 1.32(1 \mathrm{H}$, overlap $)$ & & 22.4 \\
\hline $9^{\prime}$ & 44.8 & $1.47(1 \mathrm{H}, \mathrm{m}), 1.23(1 \mathrm{H}$, overlap $)$ & & 43.7 \\
\hline $10^{\prime}$ & 34.7 & & & 33.6 \\
\hline $11^{\prime}$ & 72.8 & & & 73.0 \\
\hline $12^{\prime}, 13^{\prime}$ & 27.1 & $1.20(3 \mathrm{H}, \mathrm{s})$ & & 26.7 \\
\hline & 27.2 & $1.21(3 \mathrm{H}, \mathrm{s})$ & & 27.4 \\
\hline $14^{\prime}$ & 19.1 & $0.93(3 \mathrm{H}, \mathrm{s})$ & & 18.6 \\
\hline $15^{\prime}$ & 18.8 & $1.48(3 \mathrm{H}, \mathrm{s})$ & & 30.3 \\
\hline
\end{tabular}

Table 3) except for a small difference in the chemical shifts of the carboxyl, an oxygenated quaternary carbon, and olefinic carbons. This data indicated that $\mathbf{5}$ should be a sesquiterpene dimmer between hinokiic acid ${ }^{10)}$ and criptomeridiol. ${ }^{11)}$ These two compounds were previously isolated from the same source and their structures and NMR data assignments were fully resolved. ${ }^{4)}$ This expectation was confirmed by the upfield shift of C-12 (from $\delta_{\mathrm{C}} 171.4$ to 166.1 ) and the different olefinic carbon chemical shift at C-7 (from $\delta_{\mathrm{C}} 131.2$ to 133.5 ) and C-8 (from $\delta_{\mathrm{C}} 135.6$ to 131.4 ) related to hinokiic acid, and the downfield shift of C-4' (from $\delta_{\mathrm{C}}$ 73.1 to 85.6) and upfield shifts of C-3' (from $\delta_{\mathrm{C}} 41.3$ to 38.1) and $\mathrm{C}-15^{\prime}$ (from $\delta_{\mathrm{C}} 30.3$ to 18.8). Therefore, 5 was a sesquiterpene dimmer at C-12 of hinokiic acid and C-4' of criptomeridiol. Confirmation of the structures of the two parts were carried out by HMBC analysis as shown in Fig. 2. The relative stereochemistry of each sesquiterpene part was determined by ROESY analysis (see Fig. 2). The absolute configuration should be same as those of hinokiic acid and criptomeridiol previously isolated from the same source. Thus, the structure of $\mathbf{5}$ was determined to be ent-cryptomeridiol-4-yl-(-)-hinokiate as shown in Fig. 1. Compound 5 was stable under alkaline hydrolysis conditions. Compound 5 was reacted with $5 \% \mathrm{KOH}$ in $\mathrm{MeOH}$ under reflux for $3 \mathrm{~h}$ to recover $\mathbf{5}$ and no other product on TLC analysis. This should be based on the structure of $\mathbf{5}$, which is ester of the tertiary alcohol.

Partial cis-trans isomerizations between $\mathbf{1}$ and $\mathbf{2}$ and between $\mathbf{3}$ and $\mathbf{4}$ were observed after long term preservation at room temperature. Thus the cis or tran isomers should be artifacts through extraction and purification procedure.

\section{Experimental}

Melting points were measured with a Yanaco MP-3 micro-melting point apparatus and the temperatures were not corrected. Optical rotations were recorded on a JASCO P-1010 polarimeter at $25^{\circ} \mathrm{C}$. UV and IR spectra were recorded on a U-2001 Spectrophotometer (Hitachi) and FT-IR Spectroscope (Perkin Elmer), respectively. NMR spectra were recorded on a Bruker-DRX $\left({ }^{1} \mathrm{H}-\mathrm{NMR} ; 600 \mathrm{MHz},{ }^{13} \mathrm{C}-\mathrm{NMR} ; 150 \mathrm{MHz}\right)$ and a JEOL- $\alpha-500\left({ }^{1} \mathrm{H}-\mathrm{NMR}\right.$; $500 \mathrm{MHz},{ }^{13} \mathrm{C}-\mathrm{NMR} ; 125 \mathrm{MHz}$ ) spectrometers using $\mathrm{CDCl}_{3}$ as the solvent and tetramethylsilane (TMS) as an internal standard. HR-EI-MS data were recorded on a JEOL-HX110 mass spectrometer. Preparative and analytical HPLC were performed on reverse phase columns (Mighty sil RP-18 and 8, Kantho Chemical Co., Ltd) with $\mathrm{CH}_{3} \mathrm{CN}-\mathrm{H}_{2} \mathrm{O}$ and $\mathrm{MeOH}-\mathrm{H}_{2} \mathrm{O}$ solvent systems. Silica gel 60 (Merck) was used for column chromatography. Analytical and preparative thin layer chromatography (PLC) were carried out on precoated Kieselgel $60 \mathrm{~F}_{254}(0.25 \mathrm{~mm}$ and $0.5 \mathrm{~mm}$ thick, Merck $)$.

Plant Material The leaves of C. obtusa were collected in the mountainous northern part of Hiroshima prefecture, Japan by Mr. T. Sano of Hiroshima Prefectural Forestry Research Center in October 2003, and a voucher specimen was deposited in the Faculty of Life and Environmental Sciences, Prefectural University of Hiroshima.

Extraction and Isolation The process for the extraction and isolation was carried out as shown in our previous work. ${ }^{1)}$ Air-dried leaves of $C . o b$ tusa $(3.9 \mathrm{~kg})$ were refluxed with $\mathrm{MeOH}$ and the filtrate was evaporated to give a residue $(538 \mathrm{~g})$. The extract was suspended in water and partitioned successively with EtOAc and $n$-BuOH to afford an ethyl acetate soluble fraction $(270 \mathrm{~g})$, a $n$-BuOH soluble fraction $(251 \mathrm{~g})$ and an aqueous residue. The EtOAc fraction $(110 \mathrm{~g})$ was chromatographied on a silica gel column with a gradient $\mathrm{CHCl}_{3}-\mathrm{MeOH}$ solvent system to afford ten fractions (Fr. 1-10). Purification of some parts of the fractions was reported in our previous report. ${ }^{1)}$ Residual fraction Fr. 3 (26.5 g) was further separated by silica gel column chromatography using a hexane-EtOAc solvent system to give nine fractions (Fr. 3-1-3-9). Fr. 3-4 (4.11 g) was further purified by preparative HPLC with reverse phase columns and preparative layer chromatography (PLC) to give compounds $\mathbf{1}(37.0 \mathrm{mg}), \mathbf{2}(18.7 \mathrm{mg}), \mathbf{5}(73.0 \mathrm{mg})$. Preparative HPLC purification of Fr. 3-5 (0.50 g) gave compounds $3(46.0 \mathrm{mg})$, and 4 
(14.0 mg).

Compound 1: Colorless needles, mp 115- $117^{\circ} \mathrm{C}(\mathrm{MeOH}) .[\alpha]_{\mathrm{D}}^{21}+20.8^{\circ}$ $\left(c=0.037 ; \mathrm{CHCl}_{3}\right)$. HR-EI-MS: $m / z 434.2823[\mathrm{M}]^{+}\left(\right.$Calcd for $\mathrm{C}_{29} \mathrm{H}_{38} \mathrm{O}_{3}$, 434.2821). IR $v_{\max } \mathrm{cm}^{-1}(\mathrm{KBr}): 3340,2931,1687,1632,1606,1514$. UV $\lambda_{\max } \mathrm{nm}(\varepsilon)(\mathrm{MeOH}): 226(7184), 298(8658), 310(8623) .{ }^{1} \mathrm{H}-$ and ${ }^{13} \mathrm{C}-$ NMR spectral data, see Tables 1 and 2 .

Compound 2: Colorless amorphous solid, mp 176-178 ${ }^{\circ} \mathrm{C}(\mathrm{MeOH})$. $[\alpha]_{\mathrm{D}}^{21}-28.2^{\circ}\left(c=0.044 ; \mathrm{CHCl}_{3}\right)$. HR-EI-MS $m / z: 434.2832[\mathrm{M}]^{+}$(Calcd for $\left.\mathrm{C}_{29} \mathrm{H}_{38} \mathrm{O}_{3}, 434.2821\right)$. IR $v_{\max } \mathrm{cm}^{-1}(\mathrm{KBr}): 3372,2931,1679,1632,1605$, 1516. UV $\lambda_{\max } \mathrm{nm}(\varepsilon)(\mathrm{MeOH}): 227$ (7068), 298 (9982), $311(10798) .{ }^{1} \mathrm{H}-$ and ${ }^{13} \mathrm{C}$-NMR spectral data, see Tables 1 and 2 .

Compound 3: Colorless amorphous solid. $[\alpha]_{\mathrm{D}}-22.3^{\circ}(c=0.014$, $\mathrm{MeOH}$ ). HR-EI-MS $m / z: 450.2784[\mathrm{M}]^{+}\left(\right.$Calcd for $\left.\mathrm{C}_{29} \mathrm{H}_{38} \mathrm{O}_{4}, 450.2770\right)$. IR $v_{\max } \mathrm{cm}^{-1}(\mathrm{KBr}): 3384,2928,1694,1606,1515,1167$. UV $\lambda_{\max } \mathrm{nm}(\varepsilon)$ (MeOH): 241 (10480), 291 (8780), 323 (8080). ${ }^{1} \mathrm{H}-$ and ${ }^{13} \mathrm{C}-\mathrm{NMR}$ spectral data, see Tables 1 and 2.

Compound 4: Colorless amorphous solid. HR-EI-MS m/z: $450.2798[\mathrm{M}]^{+}$ (Calcd for $\mathrm{C}_{29} \mathrm{H}_{38} \mathrm{O}_{4}, 450.2770$ ). IR $v_{\max } \mathrm{cm}^{-1}(\mathrm{KBr})$ : 3355, 2928, 1709 , $1606,1515,1167$. UV $\lambda_{\text {max }}$ nm $(\varepsilon)(\mathrm{MeOH}): 242$ (10260), 293 (8350), 316 (8090). ${ }^{1} \mathrm{H}$ - and ${ }^{13} \mathrm{C}-\mathrm{NMR}$ spectral data, see Tables 1 and 2 .

Compound 5: Colorless needles, mp 56-58 ${ }^{\circ} \mathrm{C}(\mathrm{MeOH}),[\alpha]_{\mathrm{D}}^{21}-94.1^{\circ}$ $\left(c=0.037, \mathrm{CHCl}_{3}\right)$. HR-EI-MS m/z: $456.3586[\mathrm{M}]^{+}\left(\right.$Calcd for $\mathrm{C}_{30} \mathrm{H}_{46} \mathrm{O}_{3}$, $456.3603)$. IR $v_{\max } \mathrm{cm}^{-1}(\mathrm{KBr}): 3454,2930,1703,1650,1470,1385,1255$, 1090. UV $\lambda_{\max } \mathrm{nm}(\varepsilon)(\mathrm{MeOH}): 242(4115) .{ }^{1} \mathrm{H}-$ and ${ }^{13} \mathrm{C}-\mathrm{NMR}$ spectral data, see Table 2 .

Alkaline Hydrolysis of 1 Compound $\mathbf{1}(7.4 \mathrm{mg})$ was dissolved in $3 \%$ $\mathrm{KOH} / \mathrm{MeOH}(4 \mathrm{ml})$ and heated at $85^{\circ} \mathrm{C}$ for $3 \mathrm{~h}$. The reaction solution was poured into ice water and extracted with EtOAc. The EtOAc solution was evaporated and purified by PLC to give 1a $(2.6 \mathrm{mg})$. The aqueous fraction was acidified and extracted with $\mathrm{CHCl}_{3}$. The $\mathrm{CHCl}_{3}$ solution was evaporated to give $p$-coumaric acid $(1.5 \mathrm{mg})$. The structure of $\mathbf{1 a}$ was analyzed by NMR, HR-EI-MS spectra data, and optical rotation.

Compound 1a: Colorless amorphous solid. FAB-MS; $m / z 311[\mathrm{M}+\mathrm{Na}]^{+}$ $\mathrm{C}_{20} \mathrm{H}_{32} \mathrm{O},[\alpha]_{\mathrm{D}}^{21}-23.2^{\circ}\left(c=0.026, \mathrm{CHCl}_{3}\right),{ }^{1} \mathrm{H}-\mathrm{NMR}\left(\mathrm{CDCl}_{3}\right) \delta_{\mathrm{H}}: 0.78(3 \mathrm{H}$, $\mathrm{s}, \mathrm{Me}-20), 0.79(3 \mathrm{H}, \mathrm{s}, \mathrm{C}-19), 0.99(3 \mathrm{H}, \mathrm{s}, \mathrm{CMe}-17), 1.03(1 \mathrm{H}, \mathrm{d}, J=9.8 \mathrm{~Hz}$, $\mathrm{H}-14), 1.16(1 \mathrm{H}, \mathrm{dd}, J=11.2,2.9 \mathrm{~Hz}, \mathrm{H}-5), 1.44(1 \mathrm{H}, \mathrm{dd}, J=9.8,2.7 \mathrm{~Hz}, \mathrm{H}-$ 14), $3.11(1 \mathrm{H}, \mathrm{d}, J=11.0 \mathrm{~Hz}, \mathrm{H}-18), 3.41(1 \mathrm{H}, \mathrm{d}, J=11.0 \mathrm{~Hz}, \mathrm{H}-18), 5.45$ $(1 \mathrm{H}, \mathrm{d}, J=5.5 \mathrm{~Hz}, \mathrm{H}-15), 5.69(1 \mathrm{H}, \mathrm{d}, J=5.5 \mathrm{~Hz}) \cdot{ }^{13} \mathrm{C}-\mathrm{NMR}\left(\mathrm{CDCl}_{3}\right) \delta_{\mathrm{C}}$ : 15.6 (C-20), 17.8 (C-19), 17.9 (C-2), 19.9 (C-6), 20.2 (C-11), 24.9 (C-17), 33.2 (C-12), 35.4 (C-3), 37.0 (C7), 37.1 (C-10), 37.5 (C-4), 38.7 (C-1), 43.6 (C-13), 48.9 (C-8), 49.0 (C-5), 52.8 (C-9), 61.2 (C-14), 72.3 (C-18), 135.2 (C-15), $136.4(\mathrm{C}-16)$
Alkaline Hydrolysis of 2 Compound $2(9.0 \mathrm{mg})$ was hydrolyzed under alkaline condition to give 1a $\left\{[\alpha]_{\mathrm{D}}-19.7^{\circ}(c=0.025)\right\}(2.7 \mathrm{mg})$ and $(E)-p$ coumaric acid $(3.0 \mathrm{mg}) .(E)$-p-coumaric acid was identified with the authentic compound by TLC and HPLC.

Alkaline Hydrolysis of 3 Compound $3(15.0 \mathrm{mg})$ was dissolved in $3 \mathrm{ml}$ of $3 \% \mathrm{KOH}-\mathrm{MeOH}$ and refluxed for $30 \mathrm{~min}$. The reaction solution was poured into ice water and extracted with EtOAc to give a EtOAc solution. The EtOAc solution was evaporated and purified by PLC to give 3a (10 mg). The aqueous fraction was acidified and extracted with $\mathrm{CHCl}_{3}$ to give $(E)-p$ coumaric acid $(3 \mathrm{mg})$. ( $E$ )- $p$-Coumaric acid was identified with the authentic sample by TLC and HPLC.

3a: An amorphous solid. HR-EI-MS m/z: $304.2395[\mathrm{M}]^{+}$(Calcd for $\left.\mathrm{C}_{20} \mathrm{H}_{32} \mathrm{O}_{2}, 304.2402\right) .[\alpha]_{\mathrm{D}}-13.1^{\circ}(c=0.034, \mathrm{MeOH})$. IR $v_{\text {max }} \mathrm{cm}^{-1}(\mathrm{KBr})$ : $3429,2925,1455,1166,1047$. UV $\lambda_{\max } \mathrm{nm}(\varepsilon)(\mathrm{MeOH}): 244(8591) .{ }^{1} \mathrm{H}-$ NMR spectral data, see Table 1 and ${ }^{13} \mathrm{C}$-NMR spectral data, see Table 2 .

\section{References}

1) Xiao D., Kuroyanagi M., Itani T., Matsuura H., Udayama M., Murakami M., Umehara K., Kawahara N., Chem. Pham. Bull., 49, 1479$1481(2000)$.

2) Gao H., Wu L., Kuroyanagi M., Harada K., Kawahara N., Nakane T., Umehara K., Hirasawa A., Nakamura Y., Chem. Pham. Bull., 51, 1318-1321 (2003)

3) Kuroyanagi M., Shimomae M., Nagashima Y., Muto N., Okuda T., Kawahara N., Nakane T., Sano T., Chem. Pharm. Bull., 53, 15191523 (2005).

4) Kuroyanagi M., Ikeda R., Gao H., Muto N., Otaki T., Sano T., Kawahara N., Nakane T., Chem. Pharm. Bull., 56, 60-63 (2008).

5) Anjaneyulu A. S. R., Rao V. L., Sreedhar K., J. Nat. Prod., 65, 382385 (2002).

6) Delgado G., De Vivar A. R., Cardenas J., Pereda-Miranda R., Hueta E., Phytochemistry, 23, 2285-2288 (1984).

7) Konishi T., Konoshima T., Fujiwara Y., Kiyosawa S., J. Nat. Prod., 63, $344-346(2000)$

8) Ansell S. M., Pegel K. H., Taylor D. A. H., Phytochemistry, 32, 953959 (1993).

9) McCrindle R., Martin A., Murray R. D. H., J. Chem. Soc. (C), 1968, 2349-2354 (1968)

10) Takeshita H., Sato T., Muroi T., Ito S., Tetrahedron Lett., 10, 30953096 (1969).

11) Morita M., Nakanishi H., Morita H., Mihashi S., Itokawa H., Chem. Pharm. Bull., 44, 1603-1606 (1996). 